DOI: https://doi.org/10.46296/ig.v4i8edespdic.0050

\title{
MANTENIMIENTO CENTRADO EN LA CONFIABILIDAD (RCM) CASO DE APLICACIÓN MÁQUINA EMPACADORA DE ATÚN EN LATAS
}

\section{RELIABILITY CENTERED MAINTENANCE (RCM) APPLICATION CASE TUNA CANNING MACHINERY}

\author{
Peñafiel José ${ }^{1}$; Arteaga Ángel ${ }^{2}$; Daquinta-Gradaille Antonio ${ }^{3}$ \\ 1 Universidad Técnica de Manabí, Instituto de Posgrado. Portoviejo, Ecuador. Correo: \\ jose_luis150304@yahoo.es. ORCID ID: https://orcid.org/0000-0002-0014-0922. \\ 2 Universidad Técnica de Manabí, Carrera de Mecánica. Portoviejo, Ecuador. Correo: \\ aarteaga@utm.edu.ec. ORCID ID: https://orcid.org/0000-0002-3589-5866. \\ 3 Universidad de Ciego de Ávila "Máximo Gómez Báez". Ciego de Ávila, Cuba. Correo: \\ adaquinta@unica.cu. ORCID ID: https://orcid.org/0000-0001-7723-5324.
}

\section{Resumen}

Una línea de producción de conservas de atún en latas en la República del Ecuador es analizada, donde su programa de mantenimiento mecánico tiene como base la experiencia acumulada a través del tiempo del personal encargado, así como las recomendaciones de los fabricantes de los equipos; lo anterior representa un déficit del uso de estrategias de clase mundial en la industria de procesos. La metodología utilizada es la del Mantenimiento Centrado en la Confiabilidad ( $R C M)$, aplicada a uno de los equipos críticos de la línea de producción, como es la empacadora de atún en latas. El resultado obtenido fue el rediseño de la función mantenimiento, logrando alinear las actividades hacia la mejora del plan de mantención del equipo objeto de estudio, mediante el análisis de los modos y efectos de falla en el contexto operacional real en el que opera el mismo.

Palabras clave: Confiabilidad, criticidad, empacadora de atún, mantenimiento.

\begin{abstract}
A production line of canned tuna in the Republic of Ecuador is analyzed, determining that its mechanical maintenance program is based on the experience accumulated over the time of the personnel in charge, as well as the recommendations of the manufacturers of the machines; this represents a deficit in the use of world-class strategies in the process industry. The methodology used is Reliability Centered Maintenance (RCM), applied to one of the critical equipment of the production line, such as the canned tuna packer. The result obtained was the redesign of the Maintenance function, managing to align the maintenance activities towards improving the maintenance plan of the equipment under study, through the analysis of failure modes and effects in the real operational context in which it operates.
\end{abstract}

Keywords: Canned tuna packer, criticality, maintenance, reliability.

Información del manuscrito:

Fecha de recepción: 04 de octubre de 2021.

Fecha de aceptación: 09 de noviembre de 2021.

Fecha de publicación: 08 de diciembre de 2021. 


\section{Introducción}

A partir de la Revolución Industrial y del constante desarrollo tecnológico, se ha podido evidenciar una importante modernización de la industria de procesos; lo cual ha generado la aparición y desarrollo de diferentes tendencias y técnicas del mantenimiento industrial (Woodhouse, 2020). Lo anterior ha hecho posible que el mantenimiento industrial tenga un nuevo enfoque hacia toda la organización dentro de la industria, así como también una mayor importancia hacia aspectos de calidad, seguridad y medio ambiente, en concordancia siempre con la búsqueda de una alta disponibilidad de los equipos de la industria (Torres Valle y Figueroa del Valle, 2010).

Partiendo de la premisa de que toda actividad industrial se basa principalmente en el uso óptimo de los recursos para aumentar su productividad, el mantenimiento se considera como parte fundamental de todo proceso productivo, así como los óptimos mecanismos 0 técnicas utilizadas en dependencia del proceso y su complejidad (Luna, 2016).
En el contexto actual las actividades de mantenimiento que se desarrollan en nuestro medio, en cierta medida no han incursionado lo suficiente en la aplicación de las mejores prácticas de mantenimiento industrial, debido a que por décadas se ha venido desarrollando el mantenimiento industrial basado en planes de mantenimiento preventivo recomendados por los fabricantes y algunas actividades incorporadas gracias a la experiencia adquirida por los responsables del mantenimiento de los equipos a lo largo de su tiempo en operación; pero debido a la evolución de los equipos, los procesos y la competitividad que existe hoy en día, se evidencia la necesidad de investigar e implementar mejores prácticas y modelos de gestión de mantenimiento industrial.

Desde hace algunas décadas se han venido desarrollando varios modelos de mantenimiento industrial, siendo el Mantenimiento Centrado en la Confiabilidad (RCM) la piedra angular de la tercera generación de la evolución del mantenimiento y uno de los más difundidos y utilizados a nivel mundial; las industrias que han logrado implementarlo, han 
presentado mejoras significativas en el ámbito económico y de la confiabilidad de los equipos críticos de sus procesos productivos; lo antes mencionado se logra teniendo claro que un modelo de mantenimiento no es malo o bueno por definición y que aplicar la estrategia y los criterios técnicos adecuados juega un papel fundamental en toda gestión de mantenimiento (Gopalakrishnan et al. 2019).

El RCM se originó durante el auge de la aviación comercial en las décadas de los años 50 y 60 del siglo anterior, época en la cual dos tercios de los accidentes se debían a fallas en los equipos, lo cual dio como resultado el cambio de la perspectiva de enfocar la seguridad en los equipos. Lo antes mencionado se dio en una etapa en la que el mantenimiento era sinónimo de reparaciones periódicas rutinarias para el remplazo de piezas desgastadas (mantenimiento preventivo), pero esta práctica demostró ser poco eficaz dado los altos índices de accidentes de la época, así como ir en detrimento de los activos físicos de las compañías (Moubray, 1997).
En este punto se dio un cambio de paradigma orientado

al mejoramiento de las prácticas de mantenimiento en los activos físicos, la previsión y el manejo de fallas, surgiendo así el RCM (Ardila Marín et al. 2016).

En el Ecuador, segundo productor mundial de conservas de atún en latas después de Tailandia (Ministerio de comercio exterior, 2017; Khaliqi et al. 2019), no se evidencia un uso generalizado del modelo de mantenimiento fundamentado en el RCM; en la empresa objeto estudio, se aplica actualmente la experiencia acumulada a través del tiempo por el personal encargado del mantenimiento $\quad y \quad$ las recomendaciones de los fabricantes de los equipos, sin tomar en cuenta el empleo de estrategias de mantenimiento de clase mundial que han demostrado su efectividad como el RCM; lo anterior no asegura un índice de confiabilidad adecuado de los equipos que componen los procesos industriales.

(Quisigüiña et al. 2021) en su investigación evidenciaron la baja confiabilidad de los equipos de refrigeración de una planta 
procesadora de conservas de atún, lo cual se debe a que se aplica un modelo de mantenimiento preventivo, con base únicamente en las recomendaciones de los fabricantes y experiencia de los mecánicos y operadores de los equipos de refrigeración.

En comparación con los casos anteriores, el RCM está enfocado en promover una optimización significativa de las tareas de mantenimiento y una mejora en el aprovechamiento de los recursos destinados al mantenimiento industrial, mediante la determinación más precisa de los requerimientos de mantenimiento de los elementos físicos en su contexto operacional real (Díaz Concepción, 2016).

El mantenimiento Centrado en la Confiabilidad está ampliamente difundido y utilizado en las industrias de procesos a nivel mundial (Gopalakrishnan y Skoogh, 2018); razón por la cual, la aplicación del $\mathrm{RCM}$ en la industria atunera objeto de estudio mediante la realización del presente trabajo, se presenta como una novedosa oportunidad de resolver la problemática de esta industria; la cual a pesar de contar con un plan de mantenimiento preventivo, presenta un alto índice de fallos imprevistos que afectan directamente al rendimiento de la misma; metodología que podría aplicarse a las demás industrias del sector atunero ecuatoriano, importante sector industrial que en el año 2017 aportó con el 9\% (USD 1 092 millones) del total de las exportaciones no petroleras (Mendoza, 2018).

El objetivo del presente trabajo de investigación es rediseñar la función de mantenimiento de una empacadora de atún en latas de la industria objeto de estudio, aplicando la metodología RCM para alinear las actividades hacia la mejora del plan de mantenimiento del equipo analizado, mediante el análisis de los modos y efectos de falla en el contexto operacional real.

\section{Materiales y métodos}

La metodología a utilizar será el análisis documental a través del cual se realizará un análisis del historial de mantenimiento del equipo.

\section{Análisis histórico}

Una línea de producción de conservas de atún en latas en la República del Ecuador es analizada, 
mediante el historial de las actividades de mantenimiento de la empacadora de atún de la línea objeto de estudio, que se encuentran recopiladas en el software de mantenimiento de la empresa.

\section{Análisis de criticidad}

El Análisis de criticidad es una metodología que permite establecer la jerarquía o prioridades en instalaciones, sistemas, equipos y dispositivos; esto de acuerdo a una figura de mérito llamada "Criticidad" que es proporcional al "Riesgo", creando una estructura que facilita la toma de decisiones y el direccionamiento del esfuerzo y recursos hacia las áreas de acuerdo con su impacto en el negocio (Daquinta et al. 2018).

\section{Metodología de aplicación del RCM}

La aplicación del RCM permite determinar la criticidad de los modos de falla de un equipo dentro del proceso productivo, mediante el cálculo del número de prioridad de riesgo (NPR), siendo que ese cálculo implica conocer muy bien el funcionamiento y la dinámica de la planta (sistema) y de los diferentes equipos (subsistemas) (Montilla M. et al 2007).

$\mathrm{NPR}=\mathrm{F}^{\star} \mathrm{G}$

$F=$ frecuencia de falla

$\mathrm{G}=$ severidad de la falla

Luego de realizar el cálculo de los NPR, se puede ordenar los modos de falla de un equipo o sistema lo que permitirá pasar al proceso de toma de decisiones en cuánto a las acciones a implementar, las cuales pueden ir desde "aceptar el riesgo de la falla" hasta "rediseñar el sistema o componente", pasando por actividades de mantenimiento preventivo/predictivo dependiendo de los valores del NPR.

Para implementar un sistema de mantenimiento RCM se sugiere seguir la metodología simplificada descrita por (Montilla M. et al 2007) la cual se detalla a continuación:

Preguntas acerca del equipo y la dinámica.

La contestación adecuada a las preguntas listadas garantizará la comprensión y contextualización de la operación del equipo dentro de su entorno. 
1. ¿Cuáles son las funciones y los modelos identificados de rendimiento del recurso en el actual contexto operativo? (funciones)

2. ¿En qué formas no puede cumplir sus funciones el equipo 0 sistema? (fallas funcionales)

3. ¿En qué condiciones el equipamiento fallará? (se trata de describir los modos de falla potenciales)

4. ¿Qué ocasiona cada falla funcional? (modos de falla)

5. ¿Qué sucede cuando ocurre cada falla funcional? (efectos de la falla)

6. Con esto se describe el efecto potencial de la falla y surgen otras preguntas: ¿ocurrirá parada de la producción?, ¿ocurre reducción de la producción?, ¿la calidad del producto es afectada?, ¿Cuáles serán los daños provocados?

7. ¿Cuál es la frecuencia o probabilidad de ocurrencia de la falla? (ver tabla 1).

8. ¿Cuál es la gravedad o severidad de la falla? (ver tabla 2).

9. Una vez respondidas las preguntas anteriores se debe calcular el NPR y estimar su "Peso Relativo" (ver tabla 3).

La probabilidad de falla, representa la posibilidad de ocurrencia de un evento, la cual se determina mediante la tabla 1.

Tabla 1. Probabilidad de ocurrencia de fallas

\begin{tabular}{ccc}
\hline Rango & Probabilidad & Comentario \\
\hline $\mathbf{1}$ & $1 / 10000$ & Probabilidad remota, no se espera falla \\
$\mathbf{2}$ & $1 / 5000$ & Probabilidad baja \\
$\mathbf{3}$ & $1 / 2000$ & Probabilidad baja \\
$\mathbf{4}$ & $1 / 1000$ & Ocasional \\
$\mathbf{5}$ & $1 / 500$ & Moderada \\
$\mathbf{6}$ & $1 / 200$ & Moderada \\
$\mathbf{7}$ & $1 / 100$ & Alta \\
$\mathbf{8}$ & $1 / 50$ & Alta \\
$\mathbf{9}$ & $1 / 20$ & Muy alta \\
$\mathbf{1 0}$ & $1 / 10$ & Muy alta \\
\hline
\end{tabular}


La severidad de falla, representa el impacto que puede ocasionar la ocurrencia de los diferentes modos de falla, la cual se determina mediante la tabla 2 .

Tabla 2. Severidad de las fallas

\begin{tabular}{|c|c|c|}
\hline Rango & Probabilidad & Comentario \\
\hline 1 & Ninguno & $\begin{array}{l}\text { La falla no tendrá efecto en el ambiente, la salud, la } \\
\text { seguridad y la función del sistema }\end{array}$ \\
\hline 2 & Muy leve & $\begin{array}{l}\text { Perturbación menor del funcionamiento. Posible acción } \\
\text { correctiva durante el funcionamiento }\end{array}$ \\
\hline 3 & Leve & $\begin{array}{l}\text { Igual que la anterior pero con una acción correctiva que } \\
\text { puede durar un poco más }\end{array}$ \\
\hline 4 & $\begin{array}{l}\text { Entre leve y } \\
\text { moderado }\end{array}$ & $\begin{array}{l}\text { Perturbación menor, probabilidad de reacomodar la función } \\
\text { del sistema o demora del proceso }\end{array}$ \\
\hline 5 & Moderado & Demora del $100 \%$ del sistema o reacomodación total \\
\hline 6 & $\begin{array}{l}\text { Entre moderado y } \\
\text { alto }\end{array}$ & $\begin{array}{l}\text { Se pierde una parte importante de la función del sistema, } \\
\text { demora en la reparación }\end{array}$ \\
\hline 7 & Alto & $\begin{array}{l}\text { Alta pérdida de la función del sistema, demoras mayores } \\
\text { para restaurar su funcionamiento }\end{array}$ \\
\hline 8 & Muy alto & Se pierde función, gran demora en la reparación \\
\hline 9 & Riesgoso & $\begin{array}{l}\text { Inconvenientes graves en cuanto a seguridad, salud y } \\
\text { ambiente. La falla puede ser detectada }\end{array}$ \\
\hline 10 & Riesgoso & Igual anterior. La falla ocurrirá sin advertencia previa \\
\hline
\end{tabular}

El número de prioridad de riesgo, representa el producto de la probabilidad y la severidad, es el valor que se utiliza para priorizar las acciones de mantenimiento en orden de importancia, la cual se determina mediante la tabla 3 .

Tabla 3. Número de prioridad de riesgo

\begin{tabular}{ccc}
\hline Componente del NPR & Clasificación & Peso \\
\hline & Bajo & 1 a 5 \\
Numero de prioridad de & Medio & 5 a 10 \\
riesgo (NPR) & Alto & 10 a 20 \\
& Muy alto & 20 a 100 \\
\hline
\end{tabular}

Por medio de este análisis se realiza la toma de decisiones y define el tipo de mantenimiento o acciones a adoptar con respecto a los sistemas, 
subsistemas y componentes de las máquinas y situaciones que se pudieran presentar.

Toma de decisiones a partir del cálculo del NPR.

Una vez calculados los NPR, se deben desarrollar planes de acción para eliminar o corregir el problema potencial, básicamente se responden preguntas del estilo de ¿Qué puede hacerse para predecir o prevenir cada falla? (tareas proactivas e intervalos de labores); en este punto son de mucha ayuda los análisis causa raíz (ACR), además deben tenerse en cuenta alternativas (acciones por defecto o remediales) si una tarea proactiva adecuada no puede ser encontrada o no es económicamente factible.

La teoría del RCM hace uso de una cadena de razonamiento lógico en cuanto a la determinación del modo de proceder con respecto a los escenarios de falla encontradas, donde se llega por último a cinco alternativas posibles:

- Aceptar el riesgo de la falla.

- Instalar unidades redundantes.

- Definir actividades de mantenimiento preventivo.
- Programar actividades de mantenimiento predictivo.

- Proponer rediseño del sistema.

\section{Resultados}

\section{Aplicación del mantenimiento centrado en la confiabilidad en la industria de conservas de atún.}

A partir de la información recabada en la industria objeto de estudio, se determinó que los equipos a tomar como objeto de estudio serán las máquinas empacadoras, primero porque la calidad del producto final depende en gran medida del desempeño operativo de estos equipos y segundo que en estos equipos se evidencia la mayor cantidad de fallas, esto a pesar que cuentan con un plan de mantenimiento preventivo; ocasionando atrasos en la producción, reducción de capacidad operativa de la industria y un alto consumo de los recursos con los que cuenta el departamento de mantenimiento, debido al régimen de trabajo que conlleva la resolución constante de las consecuencia de las fallas. 


\section{Resolución de preguntas aplicadas a las máquinas empacadoras:}

\section{1.- Función principal del equipo.}

Empacar el peso indicado en la lata con una apariencia y velocidad establecida.

\section{2.- Identificación de fallas} funcionales, fallas potenciales y modos de falla. En el periodo de análisis comprendido entre los años 2017-2019 se detectaron 91 fallas funcionales en la empacadora de la línea de producción analizada, en la tabla 4 se presenta un condensado de las fallas funcionales recurrentes en el periodo de análisis.

Tabla 4. Fallas funcionales recurrentes en el periodo de análisis.

\begin{tabular}{lc}
\hline \multicolumn{1}{c}{ Modo de falla } & Total \\
\hline Bocines de pistones de transferencia desgastados & 4 \\
Bocines de brazo de pistón de compresión desgastados & 3 \\
Bocines de aplastador de túnel de ingreso desgastados & 2 \\
Leva de mando de pistones de transferencia deteriorada & 3 \\
Rodillos de teflón y pines de pistones medidores desgastados & 2 \\
Bocines de terminal de regulación de peso desgastados & 4 \\
Bocines de brazo de cuchilla desgastados & 2 \\
Correderas de mordazas desgastadas & 4 \\
Cuchillas deterioradas & 2 \\
Mangueras de aire para cilindros de pistón de compresión deterioradas & 2 \\
Bandejas de recolección de migas deterioradas & 7 \\
Pistones de transferencia deteriorados & 4 \\
Mordazas deterioradas & 5 \\
Rache de avance de bandas con falla de funcionamiento & 5 \\
Eje de salida de motor reductor desgastado & 4 \\
\hline
\end{tabular}

\section{3.- Cálculo de NPR y estimación}

de su peso. Mediante el uso de la tabla 1 se realizó un escalamiento condensado de las 91 fallas funcionales ocurridas en la máquina empacadora durante el periodo de análisis. De acuerdo a lo expuesto, en la tabla 5 se presentan las frecuencias escalas para el cálculo del número de prioridad de riesgo. 
Tabla 5. Frecuencias escaladas para cálculo de NPR.

\begin{tabular}{ccc}
\hline Rango & Frecuencia & Comentario \\
\hline 1 & $1 / 91$ & Muy baja \\
2 & $2 / 91$ & Baja \\
3 & $5 / 91$ & Ocasional \\
4 & $10 / 91$ & Moderada \\
5 & $20 / 91$ & Alta \\
6 & $50 / 91$ & Muy alta \\
\hline
\end{tabular}

Por razones de espacio se presenta en la tabla 6 el cálculo de NPR para las 5 fallas funcionales de mayor recurrencia:

Tabla 6. Cálculo de NPR.

\begin{tabular}{|c|c|c|c|}
\hline Falla funcional & $\begin{array}{l}\text { Valor asignado } \\
\text { por frecuencia }\end{array}$ & $\begin{array}{l}\text { Valor asignado por } \\
\text { severidad }\end{array}$ & NPR \\
\hline $\begin{array}{l}\text { Bandeja de recolección de migas } \\
\text { deteriorada }\end{array}$ & 4 & 1 & 4 \\
\hline Mordazas deterioradas & 3 & 8 & 24 \\
\hline $\begin{array}{l}\text { Rache de avance de bandas con falla } \\
\text { de funcionamiento }\end{array}$ & 3 & 6 & 18 \\
\hline $\begin{array}{l}\text { Bocines de terminal de regulación de } \\
\text { peso desgastados }\end{array}$ & 3 & 5 & 15 \\
\hline $\begin{array}{l}\text { Correderas de } \\
\text { desgastadas }\end{array}$ & 3 & 4 & 12 \\
\hline
\end{tabular}

\section{4.- Toma de decisiones a partir del} cálculo del NPR. Las decisiones y acciones tomadas se formularon en el siguiente orden:

\section{- Aceptación del riesgo de la falla.}

Se conservó lo mismo criterios que tienen actualmente.

- Instalación de unidad redundante. No aplica al ser equipos costosos, pero se consideró tener ciertas piezas principales de alto valor y baja rotación en el stock de repuestos, para lo cual se empleó la técnica de control de inventario basado en la planeación de mantenimiento (Zhu et al. 2020).

$\checkmark$ Tareas de mantenimiento preventivo. Las rutinas de inspección y lubricación previo al arranque diario de la producción, además de ciertos mantenimientos recomendados por el fabricante como el cambio de aceite de la caja de levas; las cuales hacen parte de las tareas 
de mantenimiento preventivo asignadas a la máquina.

$\checkmark$ Tareas de mantenimiento predictivo. La observación del desempeño diario de la máquina, análisis de vibraciones, el control de holgura de los bocines y la medición de parámetros eléctricos, son los principales factores medidos como parte del mantenimiento predictivo. $\checkmark$ Rediseño del sistema. Por el momento no se ha considerado ningún tipo de rediseño de las máquinas.

A modo de ejemplo en la tabla 7 se presenta el análisis de la falla funcional "mordazas deterioradas", donde se obtiene su criticidad, acción o decisión tomada y finalmente el mantenimiento dentro del cual quedó contemplada la atención de cada modo de falla.

Tabla 7. Análisis de falla funcional.

\begin{tabular}{|c|c|c|c|c|c|c|c|}
\hline $\begin{array}{c}\text { Falla } \\
\text { funcional }\end{array}$ & $\begin{array}{l}\text { Modo de } \\
\text { falla }\end{array}$ & $\mathbf{F}$ & $\mathbf{G}$ & Criticidad & $\begin{array}{r}\text { Acciór } \\
\text { desarrolla } \\
\text { decisión to }\end{array}$ & $\begin{array}{l}n \\
\text { da o } \\
\text { mada }\end{array}$ & Contemplado \\
\hline \multirow[t]{6}{*}{$\begin{array}{l}\text { Mordazas } \\
\text { deterioradas }\end{array}$} & $\begin{array}{l}\text { - Falta de } \\
\text { lubricación } \\
\text { de } \\
\text { correderas }\end{array}$ & 1 & 8 & 8 & $\begin{array}{l}\text { Lubricación } \\
\text { aceite } \\
\text { alimenticio }\end{array}$ & $\begin{array}{r}\text { con } \\
\text { grado }\end{array}$ & $\begin{array}{l}\text { Mantenimiento } \\
\text { autónomo }\end{array}$ \\
\hline & $\begin{array}{l}\text { - Excesivo } \\
\text { rozamiento } \\
\text { entre } \\
\text { mordazas y } \\
\text { espejo }\end{array}$ & 1 & 8 & 8 & \multicolumn{2}{|c|}{$\begin{array}{l}\text { Revisión de ajuste } \\
\text { entre mordazas y } \\
\text { espejo }\end{array}$} & $\begin{array}{l}\text { Rutinas de } \\
\text { inspección previo al } \\
\text { arranque de } \\
\text { producción }\end{array}$ \\
\hline & $\begin{array}{l}\text {-Golpe de } \\
\text { pistón de } \\
\text { trasferencia }\end{array}$ & 1 & 8 & 8 & \multicolumn{2}{|c|}{$\begin{array}{l}\text { centrado de pistón } \\
\text { de transferencia y } \\
\text { mordazas }\end{array}$} & $\begin{array}{l}\text { Rutinas de } \\
\text { inspección previo al } \\
\text { arranque de } \\
\text { producción }\end{array}$ \\
\hline & $\begin{array}{l}\text {-Excesivo } \\
\text { rozamiento } \\
\text { entre } \\
\text { mordazas y } \\
\text { cuchilla de } \\
\text { lomos }\end{array}$ & 1 & 8 & 8 & \multicolumn{2}{|c|}{$\begin{array}{l}\text { Revisión de ajuste } \\
\text { entre mordazas y } \\
\text { cuchilla de lomos }\end{array}$} & $\begin{array}{l}\text { Rutinas de } \\
\text { inspección previo al } \\
\text { arranque de } \\
\text { producción }\end{array}$ \\
\hline & $\begin{array}{l}\text {-Ajuste de } \\
\text { correderas } \\
\text { incorrecto }\end{array}$ & 1 & 8 & 8 & \multicolumn{2}{|c|}{$\begin{array}{l}\text { Revisión de ajuste } \\
\text { de correderas }\end{array}$} & $\begin{array}{l}\text { Rutinas de } \\
\text { inspección previo al } \\
\text { arranque de } \\
\text { producción }\end{array}$ \\
\hline & $\begin{array}{l}\text {-Tiempo de } \\
\text { uso }\end{array}$ & 1 & 8 & 8 & $\begin{array}{l}\text { Revisión } \\
\text { holgura } \\
\text { mordazas } \\
\text { corredera }\end{array}$ & $\begin{array}{r}\text { de } \\
\text { entre } \\
y\end{array}$ & $\begin{array}{l}\text { Mantenimiento } \\
\text { preventivo }\end{array}$ \\
\hline
\end{tabular}


Es importante destacar que si el enfoque hubiese sido trabajar estrictamente con actividades de mantenimiento preventivo, habrían aumentado las actividades del plan de mantenimiento que se ejecuta a diferentes intervalos, con el correspondiente aumento de recursos humanos y físicos, para la atención de los 6 modos de falla en estudio, sin embargo, con la aplicación del RCM se puede evidenciar que se logra una reducción significativa de la carga laboral debido a la reducción de actividades preventivas que no logran reducir la probabilidad de falla hasta un límite aceptable, reemplazándolas con rutinas de inspección y mantenimiento autónomo tal como lo muestra la tabla 8.

Tabla 8. Rutinas de inspección y mantenimiento autónomo.

\begin{tabular}{cll}
\hline Modo de falla & \multicolumn{1}{c}{ Acción o decisión } & \multicolumn{1}{c}{$\begin{array}{c}\text { Tipo de mantenimiento } \\
\text { aplicado }\end{array}$} \\
\hline 1 & Revisión operador & Mantenimiento autónomo \\
4 & Revisión diaria & Rutinas de inspección \\
1 & Aceptar la falla & Correctivo programado \\
\hline
\end{tabular}

En las tablas 9 y 10 se muestran los planes de mantenimiento previo a la aplicación de la metodología RCM y posterior a la misma.

Tabla 9. Plan de mantenimiento previo a la aplicación de la metodología RCM.

\begin{tabular}{|c|c|c|c|}
\hline \multirow[t]{4}{*}{ Mantenimiento } & Actividades principales & Frecuencia & Responsable \\
\hline & Cambio de aceite. & Semestral & Mecánico \\
\hline & $\begin{array}{l}\text { Cambio de rodamientos de cajas de } \\
\text { levas }\end{array}$ & Semestral & Mecánico \\
\hline & $\begin{array}{l}\text { Chequeo de rodamientos de rodillos } \\
\text { horizontales y verticales }\end{array}$ & Semestral & Mecánico \\
\hline \multirow[t]{3}{*}{ Preventivo } & $\begin{array}{l}\text { Revisar y lubricar con grasa grado } \\
\text { alimenticio el rache de avance de } \\
\text { bandas }\end{array}$ & Semestral & Mecánico \\
\hline & $\begin{array}{l}\text { Revisar y rectificar levas } \\
\text { desgastadas }\end{array}$ & Semestral & Mecánico \\
\hline & $\begin{array}{l}\text { Control de holgura en todos los } \\
\text { rodamientos }\end{array}$ & Semestral & Mecánico \\
\hline
\end{tabular}


Tabla 10. Reestructuración global de plan de mantenimiento, una vez aplicado RCM.

\begin{tabular}{llll}
\hline Mantenimiento & Actividades principales & Frecuencia & Responsable \\
\hline \multirow{2}{*}{ Preventivo } & $\begin{array}{l}\text { Cambio de aceite } \\
\text { Cambio de rodamientos de cajas de } \\
\text { levas }\end{array}$ & $\begin{array}{l}\text { Semestral } \\
\text { Semestral }\end{array}$ & $\begin{array}{l}\text { Mecánico } \\
\text { Mecánico }\end{array}$ \\
& $\begin{array}{l}\text { Revisión de holgura de bocines } \\
\text { Lubricación de partes móviles con } \\
\text { aceite y grasa grado alimenticio } \\
\text { Observación de desempeño }\end{array}$ & $\begin{array}{l}\text { Trimestral } \\
\text { Diario }\end{array}$ & $\begin{array}{l}\text { Mecánico } \\
\text { Operador }\end{array}$ \\
$\begin{array}{l}\text { Control de consumo eléctrico } \\
\text { Predictivo }\end{array}$ & $\begin{array}{l}\text { Diario } \\
\text { Trimestral de vibraciones }\end{array}$ & $\begin{array}{l}\text { Operador } \\
\text { Electricista }\end{array}$ \\
$\begin{array}{l}\text { Correctivo } \\
\text { programado }\end{array}$ & Embocinar, cambio de mordazas & Trimestral & Mecánico \\
\hline
\end{tabular}

Se concuerda con los autores citados en este trabajo en que la aplicación de la metodología del mantenimiento centrado en la confiabilidad permite una mejor comprensión del equipo objeto de estudio y de esta manera se puede implementar las acciones necesarias para mantener operativas las funciones para las cuales el activo fue creado.

\section{Conclusiones}

Una vez realizado el análisis de los diferentes modos de falla de la empacadora de atún en latas objeto estudio, se puede concluir que:

La aplicación de la metodología del RCM permitió rediseñar la función de mantenimiento de la empacadora de atún objeto de estudio, eliminado actividades de mantenimiento que no garantizaban valores aceptables de confiabilidad y disponibilidad, reemplazándolas por actividades de inspección y mantenimiento autónomo.

Alinear las actividades hacia la mejora del plan de mantenimiento de la empacadora de atún, mediante el análisis de los modos y efectos de falla en el contexto operacional real, conduce a la reducción de la demanda de recursos físicos y humanos.

El plan de mantenimiento propuesto para la empacadora de atún en latas, utiliza actividades de mantenimiento autónomo y preventivo, que están orientadas a la mejora de la confiabilidad y disponibilidad. 


\section{Bibliografía}

ARDILA MARÍN, J. ... [et al.] La Gerencia Del Mantenimiento: Una Revisión // Maintenance Management: A Review // GestÃo De ManutenÇÃo: Uma RevisÃo. Dimensión Empresarial, 2016, 14(2), 129.

DAQUINTA, Antonio ... [et al.] Metodología de análisis de criticidad integral de las cosechadoras de caña de azúcar CASE IH. Revista Ingeniería Agrícola, 2018, 8, 55-61.

DÍAZ, Armando ... [et al.] Implementación del Mantenimiento Centrado en la confiabilidad en empresas de trasmisión eléctrica. ingeniería mecánica, 2016, 19.

GOPALAKRISHNAN, M. ... [et al.] Machine criticality assessment for productivity improvement. International Journal of Productivity and Performance Management, 2019, 68(5), 858-878.

GOPALAKRISHNAN, M. y SKOOGH, A. Machine criticality based maintenance prioritization. International Journal of Productivity and Performance Management, 2018, 67(4), 654-672.

KHALIQI, M., T. C. PANE y R. B. M. I. FATONI Indonesian tuna position in the international market. IOP Conference Series: Earth and Environmental Science, 2019, 260, 012024.

LUNA, J. A. Introducción al mantenimiento industrial tipo TPM (Mantenimiento Productivo Total) y RCM (Mantenimiento Centrado en Fiabilidad). In. Facultad de Ingeniería y Ciencias Básicas: Politécnico Grancolombiano, 2016.

MENDOZA, M. (2018). Ecuador es una potencia atunera. lideres. Retrieved from https://www.revistalideres.ec/l ideres/ecuador-industriaprocesa-atunexportacion.html visitado el 26 agosto 2020.

\section{MINISTERIO DE COMERCIO EXTERIOR INFORME SOBRE EL SECTOR ATUNERO ECUATORIANO. 2017.}

MONTILLA M., C., J. ARROYAVE y C. SILVA Caso de aplicación de mantenimiento centrado en la confiabilidad RCM, previa existencia de mantenimiento preventivo. Scientia Et Technica, 2007, XIII (37), 273-278.

MOUBRAY, J. RCM2. Translated by S.Y.A. ELLMANN. Edtion ed. 44 Regent Street, Lutterworth, Leicestershire LE 14 4BD, United Kingdom: Aladon Ltd., 1997. 300 p. ISBN 095396032-3. 
QUISIGÜIÑA, L., Á. ARTEAGA y P.

RODRIGUEZ Determinación

de Indicadores de

Confiabilidad, Mantenibilidad

y Disponibilidad. Caso de

Estudio: Industria de

Elaboración de Conservas de

Atún. Revista Cubana de

Ingeniería, 2021, 12(2).

TORRES VALLE, A. y D. FIGUEROA DEL VALLE Diseño de ejercicio docente basado en el estudio comparativo de metodologías de optimización del mantenimiento. Ingeniería Mecánica, 2010, 13, 9-17.

WOODHOUSE, J. (2000). Introduction to the Operational Reliability". Manual de Adiestramiento, PDVSA CIED, Venezuela, pp. 1-38.

ZHU, S., W. V. JAARSVELD AND R. DEKKER Spare parts inventory control based on maintenance planning. Reliability Engineering \& System Safety, 2020, 193, 106600. 